Reprod. Nutr. Dévelop., 1988, 28 (6 B), 1773-1780

\title{
Moment de la transplantation et succès de la gestation chez les mammifères
}

Y. HEYMAN

I.N.R.A., Unité de Biologie du Développement, 78350 Jouy-en-Josas, France.

Summary. Optimum timing for embryo transfer and pregnancy rates in mammals.

In domestic mammals, the optimum time for embryo transfer greatly depends upon the species and its embryo chronology development and the technique used. Three different models (rabbit, pig, bovine) have been studied to set up the importance of cycle synchrony between donor and recipient. In the rabbit, the best survival rates were obtained after tubal transfer to highly synchronous recipients even for frozen or cultured embryos. In the pig, there was no survival difference after embryo transfer on D2 (oviduct) or D6 (uterus) and an asynchrony up to $24 \mathrm{~h}$ was possible. In the bovine, embryo transfer is usually performed at the blastocyst stage directly into the uterus. A retrospective study over 2000 recipients indicates that the highest pregnancy rates were obtained when the recipient was in heat 12 to $24 \mathrm{~h}$ before the donor.

Depuis les débuts du transfert d'embryons chez les mammifères domestiques, des progrès considérables ont déjà été réalisés et ont permis d'améliorer les taux de réussite. Chez les espèces d'intérêt zootechnique et en particulier chez les bovins, l'obtention des taux de gestation les plus élevés possibles après transplantation d'embryon est une condition essentielle pour le développement pratique et l'utilisation commerciale de cette méthode de reproduction. Parmi les nombreux facteurs qui interviennent dans le succès d'une transplantation (espèce, qualité de l'embryon, technique...). le stade du développement atteint par l'embryon et le moment du cycle de la femelle receveuse sont essentiels. L'environnement utérin varie au cours du cycle et une synchronisation entre l'« âge » de l'embryon et l'« âge » de l'utérus receveur est nécessaire chez la plupart des espèces pour obtenir une bonne survie après transplantation. Nous allons montrer, sur plusieurs modèles très différents (lapins, porcins, bovins), l'importance de cette synchronisation pour l'obtention de jeunes.

\section{Transfert tubaire ou utérin ?}

Il faut, avant tout, considérer l'espèce et son mode d'implantation.

Chez les mammifères à implantation précoce (lapin, souris, rat...), la période de «vie libre » de l'embryon entre la fécondation et la nidation est limitée et le créneau de temps pendant lequel on peut effectuer des transplantations tubaires 
ou utérines est relativement restreint ( 2 ou 3 jours). Inversement, chez d'autres espèces à implantation tardive (brebis, chèvre, bovins), la période pendant laquelle le transfert d'embryons est possible atteint ou dépasse une dizaine de jours (Betteridge et al., 1976). En fait, le choix du moment du transfert par rapport au début du cycle est étroitement lié à la technique de mise en place utilisée. Chez les petits mammifères pour lesquels l'approche chirurgicale est la seule voie d'accès au tractus génital de la receveuse, on pourra effectuer soit des transferts tubaires de zygotes en tout début de segmentation, soit des transferts utérins de morula ou blastocystes. Chez les gros mammifères (bovins) pour lesquels les techniques de prélèvement d'embryons et de transplantation par les voies naturelles (vagin, cervix) sont au point, le transfert sera exclusivement intrautérin et concernera donc des embryons plus âgés (morula, blastocystes de 6 à 8 jours chez la vache).

Chez le lapin, le moment de la transplantation a été particulièrement étudié depuis les premiers travaux de Chang (1950). Ce modèle est intéressant, car la chronologie du développement embryonnaire est bien connue chez cette espèce à ovulation provoquée. Le transit des œufs à travers l'oviducte dure 60 à $72 \mathrm{~h}$. Après transplantation synchrone dans l'oviducte de lapines receveuses, Techakumphu et al. (1987) ont montré qu'il n'y avait pas de différence de taux de survie en fonction du jour de transfert : J1 (stade 2 cellules), J2 (stade 16 cellules) ou J3 (stade morula), puisque les taux de survie sont respectivement de 68, 62 et $63 \%$. Par contre, le transfert dans l'utérus est moins favorable que le transfert dans l'oviducte, comme l'ont montré les travaux de Binkerd et Anderson (1979) et aucun embryon de $\mathrm{J} 5$ ne survit jusqu'à terme après transfert utérin (Yang et al., 1986).

\section{TABLEAU 1}

Survie embryonnaire après transplantation chez le porc

\begin{tabular}{|c|c|c|c|c|c|}
\hline & & \multicolumn{4}{|c|}{ Jour du transfert } \\
\hline & & $\mathrm{J} 2$ & $\mathrm{~J} 4$ & J5 & J6 \\
\hline $\begin{array}{l}\text { Transfert } \\
\text { synchrone }\end{array}$ & $\begin{array}{l}\text { Gestations } \\
\text { Taux de survie à J30 } \\
\text { (truies gravides) }\end{array}$ & $\begin{array}{l}25,0 \%(2 / 8) \\
63,6 \%(14 / 22)\end{array}$ & $\begin{array}{l}61,5 \%(8 / 13) \\
63,9 \%(69 / 108)\end{array}$ & $\begin{array}{l}75,0 \%(3 / 4) \\
56,7 \%(17 / 30)\end{array}$ & $\begin{array}{l}75,0 \%(6 / 8) \\
57,5 \%(42 / 73)\end{array}$ \\
\hline $\begin{array}{l}\text { Transfert } \\
\text { asynchrone }\end{array}$ & $\begin{array}{l}\text { Stade donneuse } \\
\text { Stade receveuse } \\
\text { Gestations } \\
\text { Taux de survie à J30 } \\
\text { (truies gravides) }\end{array}$ & $\begin{array}{c}\mathrm{J} 2 \\
\mathrm{~J} 1 \\
66,6 \%(2 / 3) \\
63,4 \%(26 / 41)\end{array}$ & $\begin{array}{c}\mathrm{J} 4 \\
\mathrm{~J} 3 \\
90,0 \%(9 / 10) \\
47,9 \%(58 / 121)\end{array}$ & $\begin{array}{c}\mathrm{J} 5 \\
\mathrm{~J} 4 \\
50,0 \%(2 / 4) \\
46,9 \%(15 / 32)\end{array}$ & $\begin{array}{l}- \\
- \\
-\end{array}$ \\
\hline
\end{tabular}

Résultats I.N.R.A. - Rombauts (1988).

Dans le cas des porcins, où les transplantations se font par voie chirurgicale, les taux de survie sont sensiblement les mêmes après transfert synchrone dans l'oviducte (J2) ou bien au sommet des cornes utérines (J6) (tabl. 1). Il est même 
possible de transplanter dans l'utérus des embryons plus âgés (blastocystes éclos) aux $8^{e}$ et $9^{e}$ jours du cycle (Polge, 1982). Dans ce cas, on peut indifféremment déposer les embryons dans chacune des cornes utérines ou bien dans une seule corne, en raison de la possibilité de migration trans-utérine des embryons chez cette espèce (Dziuk et al., 1964). En pratique, il semble cependant préférable d'effectuer les transferts d'embryons chez le porc soit à $\mathrm{J} 4$ (morula compactée), soit à J6 (jeune blastocyste), notamment en raison d'une meilleure évaluation de la qualité des embryons pour chacun de ces stades (Rombauts, communication personnelle).

Chez les bovins, l'embryon migre normalement dans l'utérus le $4^{e}$ jour. Tous les transferts d'embryons très précoces $(\mathrm{J} 1-\mathrm{J} 4)$ devront impérativement être faits dans l'oviducte (chirurgie), alors que le transfert dans l'utérus (cervical ou chirurgical) ne pourra être effectué qu'au-delà du $5^{e}$ jour du cycle. Cette localisation, en liaison avec le moment de la transplantation, est très importante et les expériences de Newcomb et Rowson (1975) avaient clairement démontré qu'aucune gestation n'était possible chez la vache après transplantation d'embryons précoces $(\mathrm{J} 2)$, directement dans l'utérus. En fait, l'entrée prématurée des zygotes de bovins dans l'utérus perturbe complètement le début du développement embryonnaire jusqu'à la dégénérescence (Newcomb et al., 1976).

Lorsque les embryons ont atteint le stade morula compactée ou jeune blastocyste (J6-J7), les taux de survie après transfert utérin par la voie cervicale sont de l'ordre de 60 à $65 \%$, c'est-à-dire identiques aux taux de gestations obtenus par insémination artificielle. II est possible de transplanter des blastocystes après éclosion, voire en début d'élongation, jusqu'au stade J13 (Heyman et al., 1978). La limite se situe vers le $15^{\mathrm{e}}$ jour ; à ce stade, même si la mise en place est techniquement possible, la présence de l'embryon dans l'utérus est trop tardive pour que celui-ci émette ses signaux et empêche la régression du corps jaune cyclique. Mais en pratique, chez les bovins, très peu de blastocystes sont transplantés après l'éclosion. La quasi-totalité des transplantations est réalisée à J7-J8 (jeune blastocyste), la raison essentielle étant qu'à ce stade nous sommes capables de congeler les embryons avec un bon rendement (Renard et al., 1982).

\section{Importance de la synchronisation donneuse-receveuse selon les espè-} ces.

Exemple du lapin. - Chez cette espèce, la meilleure survie est obtenue après transfert dans une receveuse parfaitement synchronisée et ceci quel que soit le stade de développement embryonnaire étudié. Une transplantation dans une receveuse ayant ovulé $24 \mathrm{~h}$ après la donneuse n'augmente pas le taux de survie, contrairement à d'autres espèces telles que souris ou hamster. Un décalage de plus de 24 h entraîne des mortalités embryonnaires très importantes avant et après implantation : le transfert de morula 65 h p.c. à des lapines receveuses en retard de 24 ou $48 \mathrm{~h}$ aboutit à des taux de survie de $51 \%$ et $1 \%$, respectivement (Techakumphu et al., 1987).

On pouvait penser que la manipulation de l'embryon in vitro (culture ou congélation) entraînait un retard de développement pouvant être compensé par 
un transfert asynchrone (receveuse en retard par rapport à la donneuse). Mais les expériences ont montré que même après culture in vitro pendant $48 \mathrm{~h}$ ou congélation à $-196^{\circ} \mathrm{C}$, les taux de survie optimum ont été obtenus après transfert synchrone (tabl. 2). Le seul cas où le transfert asynchrone $(-24 \mathrm{~h}$ ) est plus favorable concerne les embryons de lapins qui ont subi à la fois une culture in vitro et une congélation. Cette situation est voisine de ce qui est pratiqué en médecine humaine avec la congélation d'embryons obtenus par fécondation in vitro (Cohen et al., 1985 ; Mohr et al., 1985). Ces auteurs obtiennent des grossesses après transfert asynchrone d'embryons fécondés in vitro, cultivés, puis congelés. La congélation de l'embryon après une période de culture in vitro pourrait donc modifier son aptitude à se développer (reprise plus lente de l'activité métabolique et diminution des réserves endogènes). Dans ce cas, le transfert asynchrone pourrait donner à l'embryon un temps de récupération nécessaire.

TABLEAU 2

Viabilité des embryons de lapin (morula) après transfert tubaire synchrone ou asynchrone.

\begin{tabular}{|c|c|c|}
\hline \multirow{2}{*}{ Synchronie } & \multicolumn{2}{|c|}{ Taux de survie } \\
\hline & $\mathrm{Oh}$ & $-24 h^{*}$ \\
\hline Embryons frais & $63 \%(38 / 60)$ & $37 \%(25 / 68)$ \\
\hline Embryons cultivés ${ }^{* *}$ & $51 \%(53 / 103)$ & $15 \%(9 / 60)$ \\
\hline Embryons congelés & $55 \%(37 / 67)$ & $33 \%(22 / 67)$ \\
\hline Embryons cultivés + congelés & $14 \%(9 / 65)$ & $38 \%(23 / 60)$ \\
\hline
\end{tabular}

( ) Nombre de fœtus/nbre d'embryons transplantés.

* Receveuse en retard.

* Embryons prélevés à 24 h p.c. (stade 2 cellules) puis cultivés in vitro pendant 48 h jusqu'au stade morula avant transfert tubaire.

(D'après Techakumphu et al., 1987)

Exemple du porc. - Contrairement au lapin, les expériences de transfert d'embryons porcins de différents stades, dans des truies receveuses synchrones ou bien dans des truies dont le cycle est en retard de $24 \mathrm{~h}$, aboutissent à des taux de survie identiques (tabl. 1). Chez cette même espèce, les travaux de Polge (1982) ont montré qu'une désynchronisation de 2 jours est possible à condition que la receveuse soit en retard par rapport à la donneuse ( $77 \%$ de gestation). Par contre, si le décalage de 48 h est en sens inverse (receveuse en avance par rapport à la donneuse), le taux de survie chute de façon importante (9\% de gestations). L'examen des embryons récupérés seulement quelques jours après transfert révèle que les embryons transférés dans un utérus « plus âgé » dégénèrent très vite, alors que les embryons transplantés dans un utérus « plus jeune " survivent, mais avec une croissance ralentie.

Exemple de la vache. - L'influence du degré de synchronisation des cycles entre la donneuse et la receveuse a été particulièrement étudiée chez les bovins (Wright, 1981 ; Donaldson, 1985). Le degré de synchronisation nécessaire à 
l'obtention d'un taux de gestation élevé dépend étroitement de l'âge de l'embryon transplanté. Selon les observations de Kunkel et Stricklin (1978), après transfert chirurgical, les embryons les plus jeunes (morula) requièrent une synchronisation très stricte entre la venue en chaleur de la donneuse et celle de la receveuse (écart toléré : $\pm 12 \mathrm{~h}$ ). Par contre, au-delà de $\mathrm{J} 8$, le transfert utérin de blastocystes ne nécessite pas une synchronisation aussi étroite que le transfert d'embryons plus précoces.

Dans la pratique courante, les transferts d'embryons sont maintenant effectués à $\mathrm{J} 7-\mathrm{J} 8$, par voie cervicale, chez les bovins. Une analyse réalisée en 1987-1988 auprès de plusieurs unités de transplantations en France et portant sur plus de 2000 receveuses (tabl. 3 ) nous montre que les meilleurs taux de gestation sont obtenus avec des receveuses synchrones ou dont le cycle est légèrement en avance de 12 à $24 \mathrm{~h}$ (58 à $64 \%$ de gestation après transfert d'un seul blastocyste). Par contre, dès que la désynchronisation dépasse + ou $-36 \mathrm{~h}$, le taux de gestation chute de moitié ( 31 et $36 \%$ respectivement).

\section{TABLEAU 3}

Transplantation d'embryons chez les bovins : taux de gestation en fonction du degré de synchronisation receveuse/donneuse.

\begin{tabular}{lcccccccc}
\hline & \multicolumn{8}{c}{ Degré de synchronisation } \\
\cline { 2 - 8 } & $+\begin{array}{l}36 \mathrm{~h} \\
48 \mathrm{~h}\end{array}$ & $+24 \mathrm{~h}$ & $+12 \mathrm{~h}$ & 0 & $-12 \mathrm{~h}$ & $-24 \mathrm{~h}$ & $-\begin{array}{r}36 \mathrm{~h} \\
48 \mathrm{~h}\end{array}$ \\
\hline $\begin{array}{l}\text { Nombre de receveuses } \\
\text { transplantées }\end{array}$ & 16 & 74 & 230 & 1246 & 141 & 125 & 30 \\
\hline $\begin{array}{l}\text { Nombre de gestations } \\
\text { Confirmées }\end{array}$ & 5 & 48 & 144 & 731 & 77 & 65 & 11 \\
\hline$\%$ & 31,2 & 64,8 & 62,6 & 58,6 & 54,6 & 51,2 & 36,5 \\
\hline
\end{tabular}

0

$=$ Donneuse et receveuses synchrones.

$+12 \mathrm{~h}, 24 \mathrm{~h}, 36 \mathrm{~h}=$ Receveuse en chaleur avant la donneuse.

$-12 \mathrm{~h}, 24 \mathrm{~h}, 36 \mathrm{~h}=$ Receveuse en chaleur après la donneuse.

Source: Equipes françaises 1987. Transfert cervical de blastocystes frais J7-J8.

Si l'on compare aux autres espèces, il est assez curieux d'observer chez les bovins que les meilleurs taux de gestations sont obtenus lorsque la receveuse est en avance de 12 ou $24 \mathrm{~h}$ par rapport au cycle de la donneuse. En fait, il faut peut-être nuancer cette observation. Dans l'étude en question, il ne s'agit pas de receveuses en cycles naturels, mais de génisses et vaches dont l'oestrus a été induit par un traitement hormonal (progestagène ou prostaglandines). La venue en chaleur peut intervenir entre 24 et $96 \mathrm{~h}$ après l'injection de prostaglandine ou le retrait de l'implant de progestagène et les animaux qui entrent en oestrus les premiers seront mieux cyclés et sélectionnés de préférence pour être transplantés. II existe donc un biais dans le choix des receveuses pour étudier de façon rigoureuse l'influence du degré de synchronisation sur le taux de gestation. Le second point concerne le stade auquel sont effectuées les transplantations: J7-J8, l'embryon mis en place a déjà franchi l'étape critique qu'est la formation 
du blastocyste ( $7^{\mathrm{e}}$ jour). Or, après ce stade, la vitesse de multiplication cellulaire est temporairement ralentie jusqu'au J9 (Renard et Heyman, 1979). Tout se passe comme si le blastocyste subissait dès sa formation une "diapause » limitée, permettant une tolérance plus large pour le transfert. Ceci pourrait expliquer en partie que, chez la vache, une receveuse dont le cycle est légèrement en avance sur la donneuse soit plus favorable pour le développement embryonnaire et la survie. Ainsi, I'utérus receveur "précèderait» en quelque sorte la croissance exponentielle du blastocyste, qui intervient peu après $\mathrm{J} 10$ (Betteridge et Fléchon, 1988).

\section{Transfert asynchrone et mortalité embryonnaire.}

Après transfert asynchrone, l'environnement maternel est souvent inadapté au développement embryonnaire normal en raison d'un profil hormonal différent.

Chez la brebis, les expériences de transplantation ont montré que le développement normal de l'embryon dépend d'une séquence de changements dans les sécrétions utérines (Wilmut et Sales, 1981). Ces changements sont induits par les stéroïdes ovariens (Miller et Moore, 1976 ; Wilmut et al., 1985). Après transfert asynchrone chez la brebis, l'élongation du blastocyste in utero est altérée (Lawson et al., 1983), le développement est momentanément accéléré après transfert dans une receveuse en avance (embryons $\mathrm{J} 6$ dans une receveuse J9) ou au contraire ralenti dans une receveuse en retard (embryons J6 dans une receveuse J3). Dans les deux cas, les embryons ainsi perturbés ne s'implantent pas (Wilmut et Sales, 1981). Néanmoins, ce défaut d'implantation n'est pas dû à une diminution du signal embryonnaire lutéotrope : en effet, la prolongation de la phase lutéale chez la brebis receveuse, à la fois par hémi-ovariectomie et par le transfert d'un deuxième embryon parfaitement synchrone, montre que l'embryon asynchrone disparaît de l'utérus, alors que l'embryon synchrone s'implante. II existe donc une relation active entre l'âge de l'embryon et l'âge de l'utérus. Le fait qu'un utérus plus âgé stimule la croissance du blastocyste sans pour autant aboutir à l'implantation montre que l'élongation du trophoblaste et l'aptitude à mettre un signal antilutéolytique sont deux processus distincts.

Il est bien évident que le transfert désynchronisé n'est pas la seule cause de mortalité embryonnaire. Les mortalités précoces résultant d'un environnement hormonal inadéquat de la receveuse ne peuvent pas être supprimées par un apport exogène de progestérone ou d'hCG à la femelle, comme le montrent les expériences de Diskin et Sreenan (1986) chez la vache.

En conclusion, chez les mammifères domestiques, même s'il existe des différences importantes selon les espèces pour le moment optimum de la transplantation embryonnaire ou pour le degré de synchronisation entre l'âge de l'embryon et l'âge de l'utérus receveur, les contraintes sont en général bien plus strictes que pour l'espèce humaine et le macaque. Nous ne connaissons pas, parmi les mammifères domestiques étudiés, d'espèces pour lesquelles des gestations tubaires sont possibles ou bien des espèces dans lesquelles le transfert d'œufs au stade 2 cellules, directement dans l'utérus, puisse aboutir à une gestation comme c'est le cas dans l'espèce humaine. 
Chez les mammifères domestiques, la préparation des femelles receveuses joue un grand rôle dans la réussite du transfert d'embryons. Des taux de gestation élevés sont maintenant obtenus en transplantant des embryons relativement avancés (jeunes blastocystes mis en place directement dans l'utérus). Néanmoins, avec le développement des recherches sur la F.I.V. ou sur la transgenèse, il devient important de pouvoir développer des techniques relativement simples et efficaces de transfert tubaire pour des ceufs au début de leur développement. Ceci se justifie particulièrement chez les espèces pour lesquelles la culture in vitro du zygote jusqu'au stade blastocyste est particulièrement difficile et d'un faible rendement (bovins, brebis).

$27^{e}$ Réunion de la Société française pour l'Etude de la Fertilité. Paris, 29, 30 sept., $1^{\text {er }}$ oct. 1988.

Remerciements. - Nous remercions vivement I'U.N.C.E.I.A., I'O.G.E.R., I'U.R.C.E.O. et le G.I.E. Nord-Est Embryon pour leurs informations sur les transplantations d'embryons bovins, ainsi que le Dr Rombauts (I.N.R.A.) pour les données sur le porc.

\section{References}

BETTERIDGE K. J., FLEECHON J. E., 1988. The anatomy and physiology of pre-attachment bovine embryos. Theriogenology, 29, 155-187.

BETTERIDGE K. J., MITCHELL D., EAGLESOME M. P., RANDALL G. C. G., 1976 . Embryo transfer in cattle 10-17 days after estrus. VIIIth Int. Congr. on anim. Reprod. and A.I., Krakow, pp. $237-240$.

BINKERD P. E., ANDERSON G. B., 1979. Transfer of cultured rabbit embryos. Gamete Res., 2, 65-73.

CHANG M. C., 1950. Development and fate of transferred rabbit ova or blastocyst in relation to the ovulation time of recipients. J. exp. Zool., 114, 197-216.

COHEN J., SIMONS R. F., EDWARDS R. G., FEHILLY C. B., FISHEL S. M., 1985 . Pregnancies following the frozen storage of expanding human blastocysts. J. in vitro Fertil. Embryo Transfer, 2. 59-64.

DISKIN M. G., SREENAN J. M., 1986. Progesterone and embryo survival in the cow. Current Topics vet. Med. anim. Sci., 34, 142-158.

DONALDSON L. E., 1985. Matching of embryo stages and grades with recipient oestrous synchrony in bovine embryo transfer. Vet. Rec., 117, 489-491.

DZIUK P. J., POLGE C., ROWSON L. E. A., 1964. Intra-uterine migration and mixing of embryos in swine following egg transfer. J. anim. Sci., 23, 37-42.

HEYMAN Y., RENARD J. P., OZIL J. P., du MESNIL du BUISSON F., 1978 . Cervical embryo transfer at different stages in cattle, 330-335. In SREENAN J. M., Contro/ of reproduction in the cow. Martinus Nijhoff.

KUNKEL R. N., STRICKLIN W. R., 1978. Donor-recipient asynchrony, stage of embryo development and the post-transfer survival of bovine embryos. Theriogenology, 9, 96.

LAWSON R. A. S., PARR R. A., CAHILL L. P., 1983. Evidence for maternal control of blastocyst growth after asynchronous transfer of embryos to the uterus of the ewe. J. Reprod. Fert., 67, 477-483.

MILLER B. G., MOORE N. W., 1976. Effect of progesterone and estradiol on RNA and protein metabolism in the genital tract and on survival of embryos in the ovariectomized ewe. Aust. J. biol. Sci., 29, 565-573.

MOHR L. R., TROUNSON A., FREEMAN L., 1985. Deep-freezing and transfer of human embryos. J. in vitro Fertil. Embryo Transfer, 2, 1-10. 
NEWCOMB R., ROWSON L. E. A., 1975. Conception rate after uterine transfer of cow eggs in relation to synchronization of oestrus and age of the egg. J. Reprod. Fert., 43, 539-541.

NEWCOMB R., ROWSON L. E. A., TROUNSON A. P., 1976. The entry of superovulated eggs into the uterus, 1-15. In Egg transfer in cattle, EEC Symposium, Cambridge.

POLGE C., 1982. Embryo transplantation in the pig. In MERIEUX Ch., BONNEAU M., Embryo transfer in mammals, 235-242.

RENARD J. P., HEYMAN Y., 1979. Variable development of superovulated bovine embryos between D6 and D12. Ann. Biol. anim. Bioch. Biophys., 19, 1589-1598.

RENARD J. P., HEYMAN Y., OZIL J. P., 1982. Congélation de l'embryon bovin: une nouvelle méthode de décongélation pour le transfert cervical d'embryons conditionnés une seule fois en paillette. Ann. Méd. vét., 126, 23-32.

TECHAKUMPHU M., WINTENBERGER-TORRES S., SÉVELLEC C., 1987. Survival of rabbit embryos after synchronous or asynchronous transfer. Anim. Reprod. Sci. 12, 297-304.

WILMUT I., SALES D. 1., 1981. Effect of an asynchronous environment on embryonic development in sheep. J. Reprod. Fert., 61, 169-184.

WILMUT I., SALES D. I., ASHWORTH C. J., 1985. The influence of variation in embryo stage and maternal hormone profiles on embryo survival in farm animals. Theriogenology, 23, 107-119.

WRIGHT J., 1981. Non-surgical embryo transfer in cattle: embryo-recipients interaction. Theriogenology, 15, 43-56.

YANG X., SIMKIN M., BATTISTA M., WILCOX B., FOOTE R. H., 1986. Asynchronous embryo transfer in rabbit. Theriogenology, 25, 219. 